\title{
Next Generation Hemostatic Materials Based on NHS-Ester Functionalized Poly(2-oxazoline)s
}

\author{
Marcel A. Boerman, ${ }^{\dagger, \ddagger \S}$ Edwin Roozen," María José Sánchez-Fernández, ${ }^{\ddagger} \S$ Abraham R. Keereweer, ${ }^{\S}$ \\ Rosa P. Félix Lanao, ${ }^{\dagger} \S$ Johan C. M. E. Bender, ${ }^{\S}$ Richard Hoogenboom, ${ }^{\perp \odot}$ Sander C. Leeuwenburgh, ${ }^{\dagger}$ \\ John A. Jansen, Harry Van Goor, " and Jan C. M. Van Hest*, ${ }^{\dagger}$ \\ ${ }^{\dagger}$ Institute for Molecules and Materials (IMM), Radboud University Nijmegen, Heyendaalseweg 135, 6525 AJ Nijmegen, The \\ Netherlands \\ ${ }^{\ddagger}$ Department of Biomaterials, Radboudumc, Philip v. Leydenlaan 25, 6525 EX Nijmegen, The Netherlands \\ ${ }^{\S}$ GATT-Technologies BV, Toernooiveld 1, 6525 ED Nijmegen, The Netherlands \\ "Department of Surgery, Radboudumc, Geert Grooteplein 10, 6525 GA Nijmegen, The Netherlands \\ ${ }^{\perp}$ Supramolecular Chemistry Group, Department of Organic and Macromolecular Chemistry, Ghent University, Krijgslaan 281 S4, \\ 9000 Ghent, Belgium
}

\section{Supporting Information}

\begin{abstract}
In order to prevent hemorrhage during surgical procedures, a wide range of hemostatic agents have been developed. However, their efficacy is variable; hemostatic devices that use bioactive components to accelerate coagulation are dependent on natural sources, which limits reproducibility. Hybrid devices in which chain-end reactive poly(ethylene glycol) is employed as active component sometimes suffer from irregular cross-linking and dissolution of the polar PEG when blood flow is substantial. Herein, we describe a synthetic, nonbioactive hemostatic product by coating $\mathrm{N}$ hydroxysuccinimide ester (NHS)-functional poly(2-oxazoline)s (POx-NHS) onto gelatin patches, which acts by formation of covalent cross-links between polymer, host blood proteins, gelatin and tissue to seal the wound site and prevent hemorrhage during surgery. We studied different process parameters (including polymer, carrier, and coating technique) in direct comparison with clinical products (Hemopatch and Tachosil) to obtain deeper understanding of this class of hemostatic products. In this work, we successfully prove the hemostatic efficacy of POx-NHS as polymer powders and coated patches both in vitro and in vivo against Hemopatch and Tachosil, demonstrating that POx-NHS are excellent candidate polymers for the development of next generation hemostatic patches.
\end{abstract}

\section{INTRODUCTION}

One of the main challenges during surgical procedures on parenchymatous tissue is to attain control over bleeding. ${ }^{1}$ Suture control, electrocautery, ${ }^{2}$ and ultrasonic sealing ${ }^{3}$ often do not suffice during operations on for example liver or kidneys. As a result, procedures like hepatic resections ${ }^{4}$ or partial nephrectomy ${ }^{5}$ require an alternative approach to control bleeding. For this purpose, a wide range of topical hemostatic products has been developed and are clinically available. ${ }^{6-8}$

The main class of hemostatic products is composed of polymeric materials of biological origin such as starch, ${ }^{9}$ chitosan, ${ }^{10}$ oxidized regenerated cellulose, ${ }^{11,12}$ collagen, and gelatin. ${ }^{13,14}$ These biocompatible and biodegradable products accelerate the natural coagulation cascade and are available in various forms (powder, sponges, dressings). Their hemostatic action is generally limited, for example when large areas of profuse bleedings should be treated. Moreover, animal-derived products carry the risk of transmission of diseases via viral or prion agents. Within this class, Tachosil (a collagen carrier coated with human derived fibrinogen and thrombin) is considered a "gold standard" due to its widespread use in liver resection. ${ }^{13}$ However, the high costs and the use of human derived materials have stimulated the search for alternative synthetic hemostatic products. As a result, synthetic polymer sealants ${ }^{16}$ have been developed which act independently of the natural coagulation cascade by their ability to seal off the wound surface, thereby stopping the blood flow. Although the efficacy of these products is reported to be superior over naturally derived hemostats, the unknown biodegradation/ excretion profile of some of these polymers as well as their toxicity (e.g., for cyano-acrylates) ${ }^{17}$ are drawbacks of this class of materials.

A more recent approach entails the development of hybrid products, which combine the beneficial properties of both synthetic and natural polymers. Two examples are Veriset $^{18}$ (an

Received: May 14, 2017

Published: July 12, 2017 
A

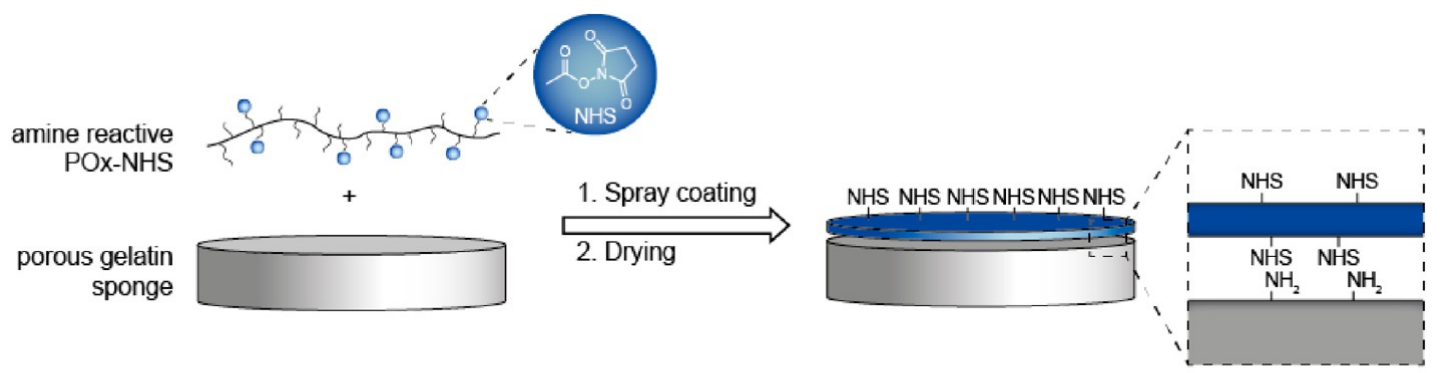

B
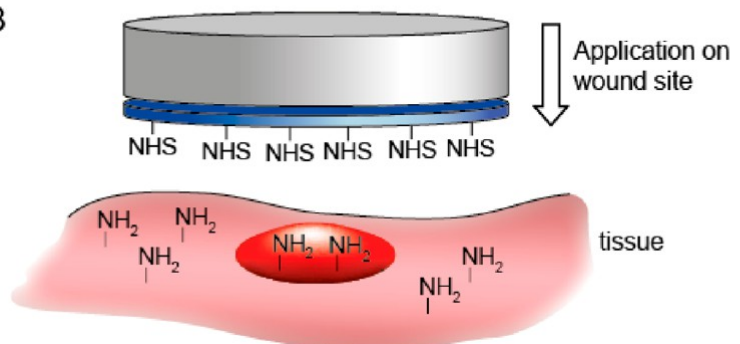

C

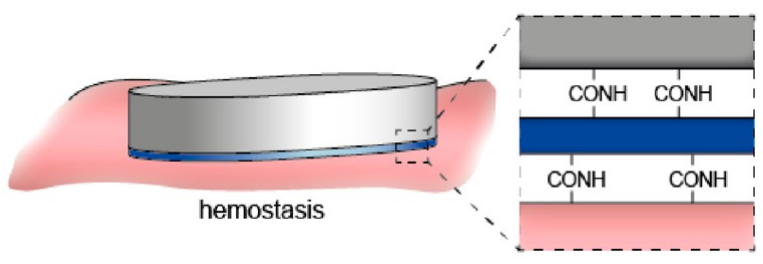

Figure 1. Schematic overview of application method and mechanism of action of poly(2-oxazoline) coated hemostatic patches. (A) Preparation of hemostatic patch by spray-coating POx-NHS onto a gelatin sponge. (B) Application of the patch onto the wound site. (C) Hemostasis is obtained by covalent cross-linking between the gelatin sponge, POx-NHS, blood proteins, and tissue in order to create a gel which seals off the wound surface and stops the bleeding.

oxidized regenerated cellulose sheet impregnated with trilysine and $\mathrm{N}$-hydroxysuccinimide ester functional 4-arm poly(ethylene glycol) (PEG-4-arm NHS)) and Hemopatch ${ }^{19-21}$ (a porous collagen carrier coated with PEG-4-arm-NHS). Both products have shown improved hemostatic efficacy compared to carriers without this coating ${ }^{21,22}$ and other commercially available products. ${ }^{18,19}$ In case of Hemopatch, the mechanism of action is based on instantaneous covalent cross-linking between PEG-4-arm-NHS and amines present in tissues, blood proteins and the collagen carrier, which seals off the wound site and allows firm fixation of the patch to the tissue. However, the intrinsically fast cross-linking of PEG-4-arm-NHS might lead to irregular sealing of the wound site (by inhomogeneous crosslinking with tissue) or poor fixation to tissue (by limited crosslinking with the collagen carrier) rendering this hemostat less effective for some surgical bleedings. Moreover, the hydrophilic nature of PEG can also cause the polymer to be flushed away from the carrier during hemorrhage, which leads to a poor hemostatic action. These potential drawbacks might be solved by modifying and fine-tuning the polymer architecture and properties. However, PEG has limited options for tailoring the degree of functionalization (only via the end groups) and polarity, which prompts further research on alternative polymers with hemostatic activity.

Poly(2-oxazoline)s (or $\mathrm{POx}^{23-26}$ ) are promising polymers for biomedical applications due to their versatile synthesis, ${ }^{27-29}$ favorable cytocompatibility, ${ }^{30-32}$ and promising excretability. ${ }^{33-37}$ In terms of polymer architecture and function, POx possesses important advantages over PEG-based systems when applied in hemostatic materials. First, cationic ring opening polymerization (CROP) allows for the introduction of both functional side chains and end groups, which is not easily achieved by anionic polymerization of PEG-based systems. Moreover, this polymerization technique allows for the synthesis of a range of copolymers, which makes it possible to accurately control the polarity and degree of side-chain functionalization of the resulting polymer.
In order to achieve optimal hemostatic performance, three main aspects of the hemostatic device should be optimized, namely, (1) the carrier, (2) the polymer coating, and (3) the coating application method onto the carrier material. As a carrier, we selected a porous gelatin sponge. Although this carrier is animal derived, it has advantages over other carrier materials, since it is fully biodegradable (4-8 weeks), shows effective uptake of blood and is already registered as a hemostatic product. ${ }^{13}$ Moreover, primary amines are available in gelatin to allow for the formation of covalent cross-links between the carrier, blood proteins, and tissue in order to create a gel that seals off the wound surface and stops the bleeding (Figure 1).

Regarding polymer design, for optimal hemostatic performance, the polymer should have sufficient reactive moieties (NHS-esters) for covalent cross-linking (e.g., with blood proteins). The polymer composition should furthermore be chosen in such a way that the polymers are soluble in water (beneficial for their biological activity) and in organic solvents (beneficial for polymer processing). Moreover, the reactive moieties should be available for cross-linking, which requires reactive side chains of sufficient flexibility and length as well as an overall polymer composition which is polar enough to allow effective wetting under physiological conditions. The crosslinking capacity should be optimized to ensure that the polymer has sufficient time to cross-link with the various components (blood, carrier, and tissue).

Regarding the coating of the hemostatic patch, we hypothesized that various parameters are important to achieve the desired hemostatic properties. First, the reactive polymer should be equally distributed over the carrier in order to obtain homogeneous hemostatic properties over the whole area of the coated patch. Second, the polymer and carrier should be combined in such a way that undesired cross-linking during the coating process is prevented. Moreover, after coating, porosity should be partially conserved in order to obtain a hemostatic patch with a dual mechanism of action of both gelatin (natural 
coagulation cascade) and the reactive polymer (sealing off the wound site by covalent cross-linking). Moreover, the blood uptake of the coated patches should be satisfactory to allow for cross-linking with all patch components (gelatin, reaction polymer, blood, and tissue), but also resistant enough to prevent excessive blood flow through the patch.

In this work, we demonstrate a versatile strategy for the preparation of a poly(2-oxazoline) based hemostatic device. First, a series of NHS-ester functionalized POx (POx-NHS) with different ratios of NHS esters and polar groups was synthesized. We studied the capacity for covalent cross-linking between these polymers and whole blood (hemostatic performance) in order to correlate the hemostatic performance with the polarity of the polymers (measured by contact angle measurements). With the preselected polymers, we utilized a spraying procedure to create a series of homogeneously coated patches. The coated patches were tested in vitro, for, for example, blood uptake and cross-linking ability. The bestperforming patches in these tests were selected to demonstrate in vivo efficacy in a compromised liver and spleen injury model of profuse bleedings in heparinized pigs.

\section{MATERIALS AND METHODS}

2.1. Materials. Gelatin sponges (Gelita Rapid, origin: porcine, $5 \times$ $8 \times 0.2 \mathrm{~cm})$ were obtained from Gelita Medical. Hemopatch was obtained from Baxter (Deerfield, IL, U.S.A.). Tachosil was obtained from Takeda (Linz, Austria). Pentaerythritol tetra(succinimidyloxysuccinyl) poly(ethylene oxide) (PEG-4-arm NHS) was obtained from NOF America corporation. Heparinized human whole blood was obtained from Sanquin (Nijmegen, The Netherlands).

2.2. Synthesis. Experimental procedures for the synthesis of P1P7 can be found in the Supporting Information.

2.3. Gelation Test. This experiment was performed using an inverted vial test adapted from literature. ${ }^{38}$ Polymer powders $(20 \mathrm{mg})$ were mixed with freshly obtained heparinized human whole blood (1 $\mathrm{mL}$ ) in a glass vial and vortexed until a visible gel was formed (gelation time).

2.4. Contact Angle Measurements. Microscope coverslips (2 $\mathrm{cm}^{2}$ ) were soaked in absolute ethanol, sonicated (30 s) and dried under reduced pressure for $15 \mathrm{~min}$. Polymer films were prepared by spin-coating the polymer solutions $(15 \mathrm{mg} / \mathrm{mL}$ in DCM, $1 \mathrm{~mL})$ onto the microscope coverslips (12000 rpm, $30 \mathrm{~s})$ using a Spin $150 \mathrm{spin}-$ coater. Subsequently, the coated slides were dried overnight under reduced pressure. Static contact angles were measured on an OCA-20 goniometer. For each measurement, $1 \mu \mathrm{L}$ of doubly distilled water was placed onto the spin-coated films at room temperature. The spreading of the droplet was imaged using a high speed video camera using 1 frame/second for $30 \mathrm{~s}$. The contact angle was determined based upon the Laplace Young fitting using the imaging software provided by the supplier (SCA 20, version 2.1.5 build 16). To determine the contact angle, the first representative frame in which a drop shape was observed was selected for analysis. The measurements were conducted in triplo per sample $(n=3)$.

2.5. Coating Deposition. Spray coating was performed using an Exactacoat spraying machine (Sono-Tek) equipped with an Accumist ultrasonically agitated nozzle. Coating was performed at a dispensing rate of $1 \mathrm{~mL} / \mathrm{min}$, a pressure of $40 \mathrm{mbar}$ and a coating speed of 40 $\mathrm{mm} / \mathrm{s}$, by moving both in the $x y$-direction over a programmed area. The nozzle height was set $30 \mathrm{~mm}$ from the top of the substrate. The coating density was adjusted by spraying multiple layers of polymer (coating cycles $(n)$ ) onto the substrate. Polymer solutions were prepared in 2-butanone/2-propanol $(\mathrm{v} / \mathrm{v}, 1: 1)$ with a final polymer concentration of $90 \mathrm{mg} / \mathrm{mL}$. After coating, the patches were dried in a vacuum oven $\left(50 \mathrm{mbar}, 50{ }^{\circ} \mathrm{C}\right)$. The coating density $\left(\mathrm{mg} / \mathrm{cm}^{2}\right)$ was determined by weighing the patches (before (carrier) and after coating $($ carrier + polymer $)(\mathrm{mg}))$ divided by the coated area $\left(\mathrm{cm}^{2}\right)$.
2.6. Scanning Electron Microscopy. Samples were attached to an aluminum holder by conducting carbon tape. Afterward, these samples were sputter coated using a gold/palladium coater (Cressington $208 \mathrm{HR})$ for $30 \mathrm{~s}(80 \mathrm{~mA})$. At different magnifications, images were acquired at an accelerated voltage of $3 \mathrm{kV}$ using a JEOL 6330 Cryo Field Emission Scanning Electron Microscope (SEM).

2.7. Blood Uptake. This experiment was performed using a procedure adapted from literature. ${ }^{13}$ Coated gelatin patches with different coating densities $\left(0,3,6\right.$, and $\left.9 \mathrm{mg} / \mathrm{cm}^{2}\right)$ were weighed ("dry weight" (mg)) and soaked into a mixture of heparinized blood/PBS (v/v, 1:1) with the coated side facing the blood mixture. The patches were allowed to absorb blood for $30 \mathrm{~s}$. After this, superficial blood was removed using a filter paper and the patches were weighed again ("weight after" (mg)) and the amount of absorbed blood ("blood" $(\mathrm{mg})$ ) was determined (wt after $(\mathrm{mg})$ - wt before $(\mathrm{mg})$ ). The blood uptake was defined by calculating the amount of absorbed blood per $\mathrm{g}$ patch. The measurements were performed in 6-fold for each sample $(n$ $=6$ ). Significant differences between samples were analyzed using ANOVA followed by a post hoc Tukey-Kramer multi comparison test.

2.8. Adhesion Test. This experiment was performed and designed according to modified ASTM F2258-05 standards. ${ }^{39}$ The samples were attached with double sided tape to 3D-printed grip tabs of $2 \mathrm{~cm}^{2}$ and these tabs were placed into a single column tensile tester (Z2,5,Zwick/Roell, Ulm, Germany, containing a $20 \mathrm{~N}$ load cell). Heparinized blood $(200 \mu \mathrm{L})$ was put between the coated patches which were pressed down using a weight of $20 \mathrm{~g}$. The patches were allowed to cross-link with blood for defined times $(1,5$, and $15 \mathrm{~min})$. Subsequently, the patches were pulled apart and the load at failure $\left(F_{\max } N\right)$ was measured. The measurements were performed in 6-fold for each sample $(n=6)$. Significant differences between samples were analyzed using ANOVA followed by a post-hoc Tukey-Kramer multicomparison test.

2.9. In Vivo Efficacy Test. Heparinized (10k units) pigs ( $n=4,30$ $\mathrm{kg}$ ) were used in this study. Permission for this experiment was granted by the responsible ethical committees at the Ministry of Education of the Czech Republic (Project \#56-2015/processing \#MSMT-42725/2015-6) Surgery was performed using standard aseptic techniques. A midline laparotomy was performed to access liver and spleen. Using a biopsy punch, standardized lesions were created in liver and spleen ( $8 \mathrm{~mm}$ diameter, $3 \mathrm{~mm}$ deep $)$. Hemostatic patches of $2.7 \times 2.7 \mathrm{~cm}$ were used in this study $(n=8$ per prototype randomized per organ using a balanced latin square). After the lesion was created, the blood flow was assessed according to a visual scoring system $(0=$ no bleeding, $0.5=$ oozing, $1=$ very slight, $2=$ slight, $3=$ moderate, $4=$ severe) described in literature. ${ }^{40}$ Afterward, superficial blood was removed using a dry gauze. Subsequently, the patches were applied with the coated side facing the organ and digital pressure was applied for $1 \mathrm{~min}$ using a dry gauze. The efficacy of the patches was evaluated after 0,1 , and $5 \mathrm{~min}$ by monitoring the bleeding (yes or no). Successful hemostasis was achieved if no bleeding was observed after 5 min without pressure (yes or no). Additionally, after $5 \mathrm{~min}$, the bleeding was scored according to the scoring system and the adhesion of the patch to the organ was tested.

2.10. Statistics. Statistical analyses were conducted using GraphPad Instat software. All results were reported as mean \pm standard deviation. Differences among groups were analyzed by ANOVA using a Tukey-Kramer Multi comparison test and $p$-values of 0.05 or lower were considered as significantly different.

\section{RESULTS AND DISCUSSION}

3.1. Synthesis. In order to create poly(2-oxazolines) with the desired characteristics for application as reactive coating in a hemostatic patch, both polarity and reactivity had to be optimized. We selected NHS-esters as the reactive moieties in view of their reactivity toward primary amines and their routine application in related medical devices. ${ }^{7}$ Since direct incorporation of NHS-esters as functional group is not compatible with cationic ring opening polymerization (CROP), we used methyl 
Scheme 1. Synthesis of NHS-Ester Functionalized Polymers (POx-NHS; P1-P7) ${ }^{a}$

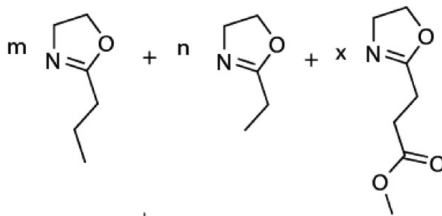

i)

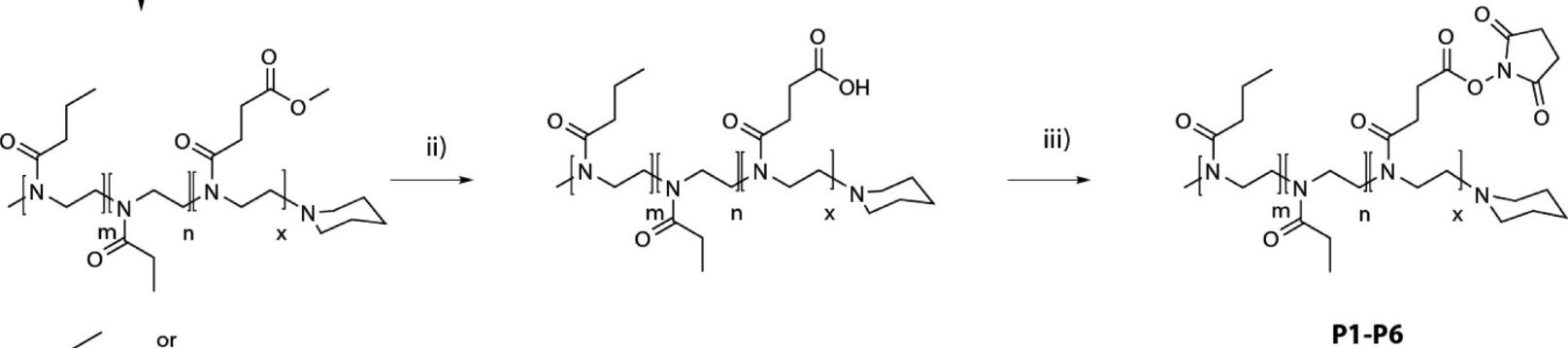<smiles>[Y]C(CCNC(C)(C)NCCO)(NC(=O)CCC(=O)OC)N(C)CCO</smiles>

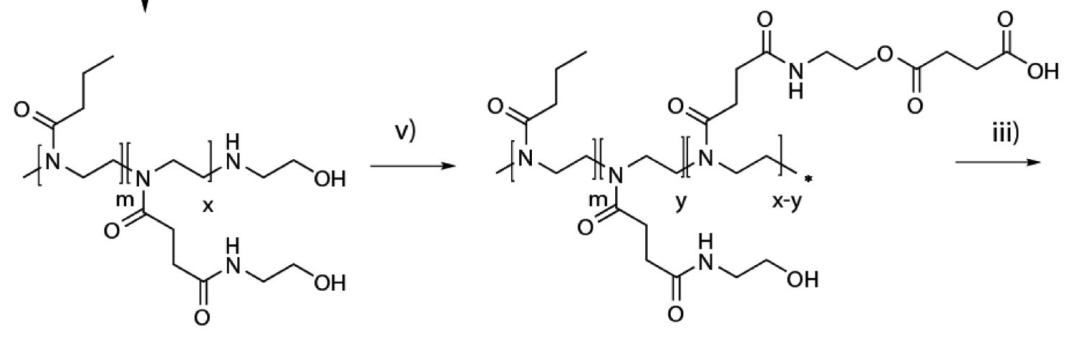

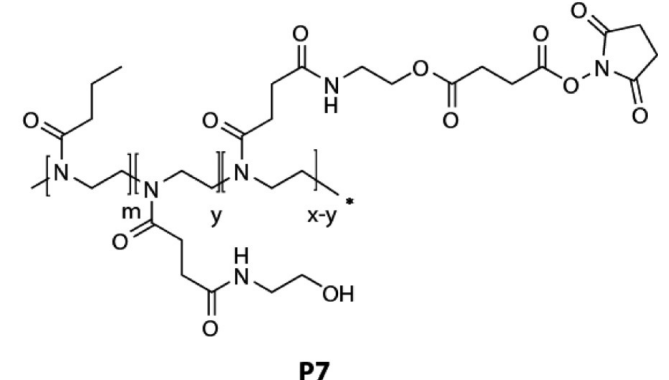

${ }^{a}$ Reagents and conditions: (i) methyl tosylate, $140{ }^{\circ} \mathrm{C}, \mathrm{CH}_{3} \mathrm{CN}$, (ii) $0.1 \mathrm{M} \mathrm{NaOH}$, rt, (iii) NHS-OH, DIC, DCM, rt, (iv) 2-amino-ethanol, $60{ }^{\circ} \mathrm{C}$, 300 mbar, (v) succinic anhydride, DMAP, DMF/DCM (v/v, 1:9, rt).

Table 1. Analytical Data of Synthesized Polymers (P1-P7)

\begin{tabular}{|c|c|c|c|c|c|c|c|c|c|}
\hline \multirow[b]{3}{*}{$\#$} & \multirow[b]{3}{*}{ polymer } & \multirow[b]{3}{*}{$m / n / x$} & \multicolumn{4}{|c|}{$\%$ funct. $\left({ }^{1} \mathrm{H}\right.$ NMR $)$} & \multirow{3}{*}{$\frac{\mathrm{UV}}{\mathrm{NHS}}$} & \multirow{2}{*}{\multicolumn{2}{|c|}{$M_{\mathrm{n}}(\mathrm{kg} / \mathrm{mol})$}} \\
\hline & & & \multirow{2}{*}{$\frac{m}{n \text { PropOx }}$} & \multicolumn{2}{|c|}{$n$} & \multirow{2}{*}{$\begin{array}{c}x \\
\text { NHS }\end{array}$} & & & \\
\hline & & & & EtOx & $\mathrm{OH}$ & & & $\mathrm{SEC}^{a}$ & $\boxplus^{a}$ \\
\hline P1 & $\mathrm{P}(n$ PropOx-c-NHS $)$ & $90-0-10$ & 90 & & & 10 & 11 & 12.6 & 1.15 \\
\hline $\mathrm{P} 2$ & $\mathrm{P}(n$ PropOx-c-NHS $)$ & $75-0-25$ & 71 & & & 29 & 26 & 13.9 & 1.11 \\
\hline P3 & $\mathrm{P}(n$ PropOx-c-EtOx-NHS $)$ & $40-50-10$ & 40 & 49 & & 11 & 9 & 12.4 & 1.18 \\
\hline P4 & $\mathrm{P}(n$ PropOx-c-EtOx-NHS $)$ & $40-35-25$ & 40 & 36 & & 24 & 22 & 12.4 & 1.26 \\
\hline P5 & $\mathrm{P}(n$ PropOx-c-EtOx-NHS $)$ & $50-40-10$ & 49 & 40 & & 11 & 11 & 12.3 & 1.16 \\
\hline P6 & $\mathrm{P}(n$ PropOx-c-EtOx-NHS $)$ & $50-25-25$ & 50 & 26 & & 24 & 23 & 14.6 & 1.18 \\
\hline P7 & $\mathrm{P}(n$ PropOx-c-OH-NHS $)$ & $70-10-20$ & 70 & & 15 & 15 & 15 & 18.8 & 1.25 \\
\hline
\end{tabular}

ester functionalized 2-methoxycarbonylethyl-2-oxazoline (MestOx) as monomer instead. This group can be easily modified after the polymerization by direct amidation ${ }^{41}$ or hydrolysis, ${ }^{42-44}$ as has been described in literature. Furthermore, it has been efficiently copolymerized before with various comonomers (including 2-ethyl-2-oxazoline $(\mathrm{EtOx})^{45}$ and 2$n$-propyl-2-oxazoline $\left.(n \text { PropOx })^{46}\right)$. For the synthesis of the various POx-NHS (P1-P7) we used two different synthetic routes, as depicted in Scheme 1. In all cases, polymers were synthesized by CROP of different ratios of EtOx, $n$ PropOx, and MestOx under inert atmosphere using microwave conditions, ${ }^{47}$ yielding both $n$ PropOx-MestOx and $n$ PropOx-EtOx-MestOx copolymers. In the first route, the MestOx groups were hydrolyzed $(0.1 \mathrm{M} \mathrm{NaOH})$, resulting in a copolymer containing carboxylic acid moieties, which were subsequently activated with $N$-hydroxysuccinimide yielding P1-P6. In the second route, MestOx was postmodified by an amidation reaction with ethanol amine, yielding copolymers equipped with a hydroxyl 
Table 2. Overview of Hemostatic Performance (P1-P7)

\begin{tabular}{|c|c|c|c|c|c|}
\hline \# & polymer & $\% \mathrm{NHS}$ & functional group content ${ }^{a}$ (mmol NHS/g polymer) & contact angle $^{b}(\mathrm{deg})$ & gelation time $e^{c}(\mathrm{~min})$ \\
\hline P1 & $\mathrm{P}(n$ PropOx-c-NHS $)$ & 10 & 0.76 & 36 & 6 \\
\hline $\mathbf{P 2}$ & $\mathrm{P}(n$ PropOx-c-NHS $)$ & 29 & 2.19 & 57 & 6 \\
\hline P3 & $\mathrm{P}(n$ PropOx-c-EtOx-NHS $)$ & 11 & 0.89 & 26 & 3 \\
\hline P4 & $\mathrm{P}(n$ PropOx-c-EtOx-NHS $)$ & 24 & 1.59 & 24 & 3 \\
\hline P5 & $\mathrm{P}(n$ PropOx-c-EtOx-NHS $)$ & 11 & 0.88 & 23 & 3 \\
\hline P6 & $\mathrm{P}(n$ PropOx-c-EtOx-NHS $)$ & 24 & 1.57 & 21 & 3 \\
\hline P7 & $\mathrm{P}(n$ PropOx-c-OH-NHS $)$ & 15 & 0.91 & 23 & 1 \\
\hline \multicolumn{6}{|c|}{ Controls } \\
\hline P8 & $\mathrm{P}(\mathrm{EtOx})$ & & & 26 & no gel \\
\hline P9 & $\mathrm{P}(n$ PropOx $)$ & & & 57 & no gel \\
\hline P10 & mPEG-OH & & & 24 & no gel \\
\hline P11 & PEG-4-arm-NHS ${ }^{d}$ & & 0.36 & 21 & instantaneous \\
\hline
\end{tabular}

${ }^{a}$ Calculated using NHS-content, which was determined by ${ }^{1} \mathrm{H}$ NMR spectroscopy. ${ }^{b}$ The measurements were conducted in triplo, blank measurement glass slide $\left(66^{\circ}\right){ }^{c}$ The gelation was determined by the inverted vial method. ${ }^{d}$ Obtained from commercial source.

moiety in the side chain. Subsequently, these hydroxyl groups were partially converted to carboxylic acid moieties using succinic anhydride, which were subsequently modified into reactive esters by coupling with $N$-hydroxysuccinimide (P7). Importantly, this second route installs a hydrolytically sensitive group in the side chain, favorable for degradation. As listed in Table 1, polymers P1-P7 were synthesized with good control over the ratio of functional groups, number-average molar mass and dispersity values. The various synthesized polymers (P1P7) were analyzed with regard to the amount of NHS groups present using both ${ }^{1} \mathrm{H}$ NMR and UV-vis spectroscopy, confirming a good agreement between the theoretical and experimental compositions.

3.2. Hemostatic Performance. As a first screening for hemostatic activity, the POx-NHS polymers were brought in contact with human whole blood and the formation of gels by mixing polymers with blood was analyzed using the inverted vial test. Besides the POx-NHS series, negative controls (polymers without NHS ester) were tested as well. In addition, a benchmark polymer (PEG-4-arm NHS used in Hemopatch) was included as a positive control to compare functional group density (mmol NHS/g polymer) in relation to the usage of different polymers. The results of these tests are listed in Table 2. As expected, polymers with NHS-esters (P1-P7) gelled with blood due to the presence of the amine-reactive NHS-esters groups, unlike the negative controls (P8-P12), which did not. Gelation times of the POx-NHS series (P1-P7) varied between $1 \mathrm{~min}(\mathbf{P} 7)$ to $6 \mathrm{~min}(\mathbf{P 1}-\mathbf{P 2})$, which was slower than the PEG-4-arm NHS benchmark polymer, which formed a gel with blood instantaneously.

As polarity was anticipated to be an important feature of the hemostatic capacity of the polymers, POx films were spincoated on glass slides after which static contact angle measurements were performed. ${ }^{48}$ Based on these contact angle measurements, it can be concluded that polymers functionalized with hydrophilic groups ( $\mathrm{PEG}, \mathrm{OH}$ or EtOX) (P3-P8 + P10 and P11) exhibit contact angles in a similar hydrophilic range $\left(21-26^{\circ}\right)$, while polymers without hydrophilic groups (P1 and P2 + P9) have higher contact angles, thereby making a clear difference between hydrophilic and somewhat more hydrophobic copolymers. It was further calculated that the PEG-based control (P11) shows a much lower content of NHS-functional groups $(0.36 \mathrm{mmol} / \mathrm{g}$ polymer) compared to $\mathbf{P 1 - P 7}$, with values ranging from 0.76 $\mathrm{mmol} / \mathrm{g}$ polymer for $\mathbf{P} \mathbf{1}$ to $2.19 \mathrm{mmol} / \mathrm{g}$ polymer for $\mathbf{P} 2$, which is a direct result of the limited functionalization possibilities of PEG via the end groups.

From both tests, it can be concluded that NHS-esters are essential for the formation of chemical cross-links with blood proteins. However, having a surplus of NHS-esters does not result in faster gelation. POx-prototypes which contain both NHS-esters and hydrophilic groups show faster gelation (P3P7) compared to polymers without hydrophilic groups (P1 and P2), but slower than PEG-4arm-NHS (P11), which crosslinked instantaneously. It was observed that the fast-gelating polymers also exhibited low contact angles. The difference in gelation speed between PEG-4-arm NHS and POx-NHS prototypes could, however, not be explained from the contact angle measurements. We assume that polarity and mobility of the polymer chains (limited by the spacer length between the polymer backbone and NHS-ester groups) are important parameters. PEG-4-arm NHS shows the fastest gelation, since the NHS-ester groups are highly mobile because of their attachment to the hydrophilic chain ends of the PEG-polymer. Within the POx-NHS samples, P7 shows the fastest gelation (1 min) because it has a longer spacer between the NHS group and the polymer backbone compared to P1-P6. Finally, the differences between P1-P6, with the same spacer length, can be explained because of polarity of the polymers; polymers containing hydrophilic EtOx groups (P3-P6) show gelation within $3 \mathrm{~min}$, while polymers without these groups (P1 and P2) show gelation around $6 \mathrm{~min}$. Due to its fast gelation, we selected $\mathrm{P}(n$ PropOx-OH-NHS) (P7) as the main candidate for further development of hemostatic patches.

3.3. Spray Coating Deposition. To cover the gelatin carrier with a polymer (P7) coating, a procedure was required that would result in a homogeneous polymer layer without compromising the beneficial properties of the gelatin carrier in terms of, for example, blood uptake capacity. Therefore, we used an ultrasonic spraying technique to deposit the polymer from volatile organic solvents of low toxicity onto the gelatin sponge and tune the amount of polymer by coating multiple layers (coating cycles) followed by drying the coated patches in a vacuum oven.

Using this approach, hemostatic patches (G1-G4) were prepared at various coating densities $\left(0-9 \mathrm{mg} / \mathrm{cm}^{2}\right)$ using a polymer solution of P7 $(90 \mathrm{mg} / \mathrm{mL}$ in 2-propanol/2-butanone $(\mathrm{v} / \mathrm{v}, 1: 1))$. We observed a linear relationship between the coating density $\left(\mathrm{mg} / \mathrm{cm}^{2}\right)$ and the amount of coating cycles (Table 3 ). Additionally, the coated patches were analyzed by 
Table 3. Coating and Functional Group Densities of Patches Prepared with P7 (G1-G4)

\begin{tabular}{|c|c|c|c|c|c|}
\hline \multirow[b]{2}{*}{ samples } & \multicolumn{4}{|c|}{ coating density $\left(\mathrm{mg} / \mathrm{cm}^{2}\right)$} & \multirow[b]{3}{*}{$\begin{array}{c}\text { functional group } \\
\text { density } \\
\left(\mu \mathrm{mol} \mathrm{NHS} / \mathrm{cm}^{2}\right)\end{array}$} \\
\hline & \multirow[t]{2}{*}{ theoretical } & \multicolumn{3}{|c|}{ measured } & \\
\hline \# & & mean & st dev & $n$ & \\
\hline G1 & 0 & & & & \\
\hline G2 & 3 & $3.06^{a}$ & 0.01 & 3 & 2.80 \\
\hline G3 & 6 & $5.71^{a}$ & 0.13 & 9 & 5.18 \\
\hline G4 & 9 & $9.22^{a}$ & 0.01 & 3 & 8.36 \\
\hline $\begin{array}{l}\text { Hemopatch } \\
\text { (PEG) }\end{array}$ & & $16.8^{b}$ & 2.2 & 5 & 5.38 \\
\hline
\end{tabular}

${ }^{a}$ Mass difference before (gelatin) and after coating (gelatin $+\mathrm{POx}-$ NHS). ${ }^{b}$ Determined by extraction of the polymer with DCM.

scanning electron microscopy (SEM; Figure 2), which revealed that the pores of the carrier were not sealed by the polymer coating after applying up to six coating cycles. Furthermore, the coating was homogeneously spread onto the carrier material, unlike Hemopatch (based on 4-arm-PEG), which showed a heterogeneous coverage revealing PEG-coated and uncoated domains. The analytical data of G1-G4 are summarized in Table 3. Importantly, as POx is functionalized with a higher number of NHS-esters than PEG, a lower amount of polymer was required $\left(5.7 \mathrm{mg} / \mathrm{cm}^{2}\right.$ for G3) in order to obtain a similar functional group density as Hemopatch $(\sim 5.2 \mu \mathrm{mol} \mathrm{NHS} /$ $\mathrm{cm}^{2}$ ), which is beneficial if an open, porous structure is required for the carrier material.

3.4. In Vitro Tests. 3.4.1. Blood Uptake. The blood uptake of the different POx-NHS coated patches (G1-G4) and Hemopatch was evaluated by soaking the patches (with the coated side in contact with blood) in a mixture of blood/PBS for $30 \mathrm{~s}$ and determining the blood uptake by weighing the carriers before and after the soaking process (Figure 3A). It was observed that the uptake capacity of the patches was reduced
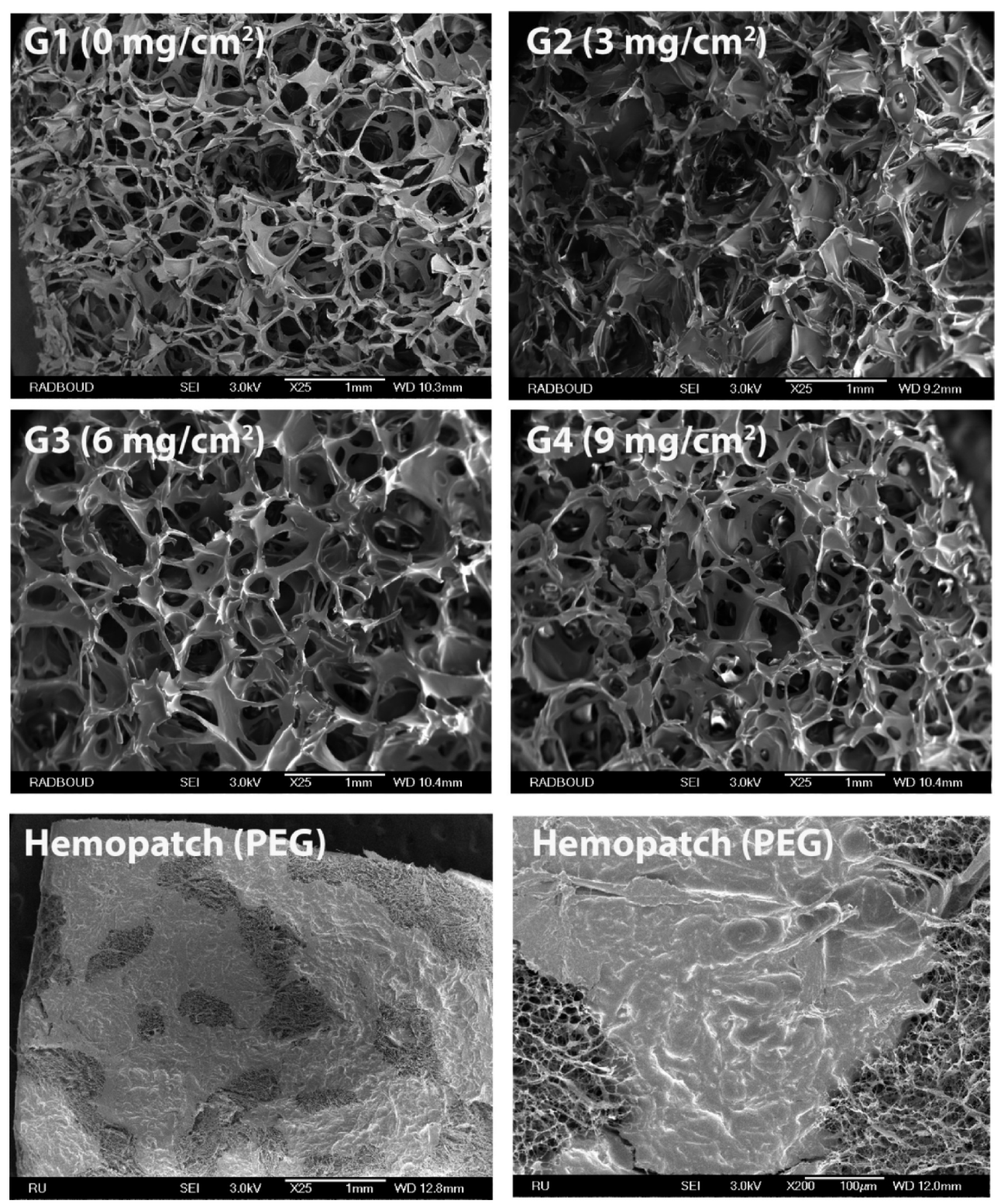

Figure 2. SEM images of POx-NHS coated patches (G1-G4) and Hemopatch (PEG). Scale bars correspond to $1 \mathrm{~mm}$ or $100 \mu \mathrm{m}$ (bottom right picture). 
A

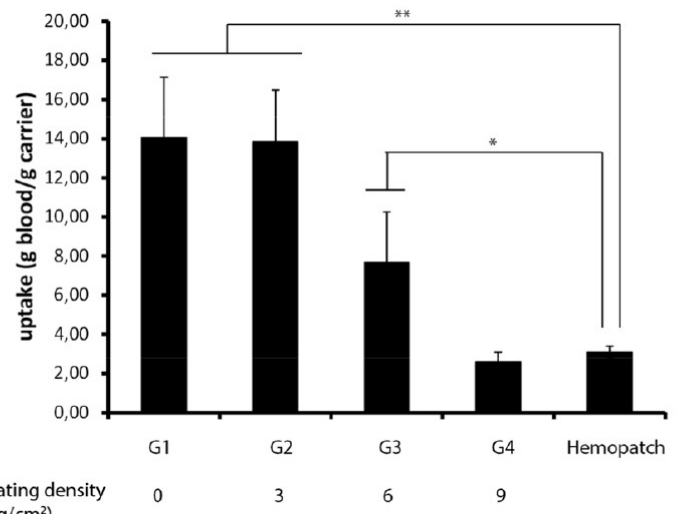

$\mathrm{B}$

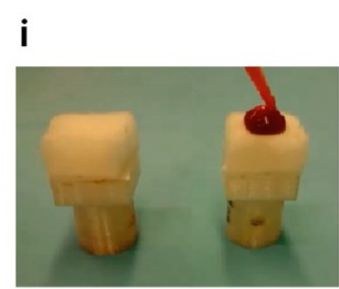

iv

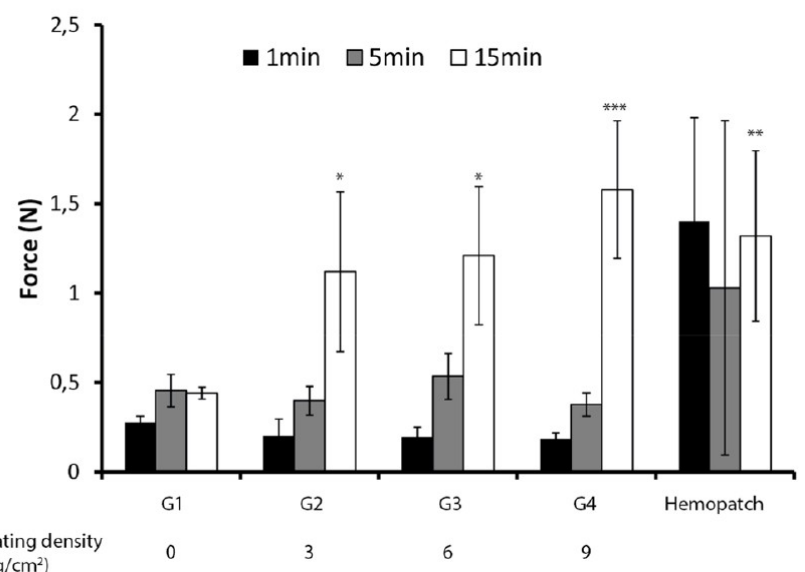

Coating densit

0

3 ii

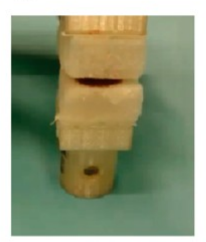

$t=1,5,15$ min iii

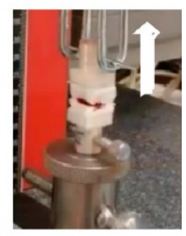

Figure 3. (A, B) In vitro tests. (A) Blood uptake capacity as a function of coating density $(* * P<0.001, * P<0.01)$; (B) In vitro adhesion test: (i) blood was applied between the patches, (ii) the patches were allowed to cross-link for defined time points ( $\mathrm{t} 1, \mathrm{t} 5, \mathrm{t} 15 \mathrm{~min}$ ), (iii) the samples were placed in a Zwick Roell tensile bench and a vertical force was applied until failure, (iv) results of the adhesion test $(* P<0.05$, $* * P<0.01, * * * P<0.001)$.

with increasing coating density. Although the polymer coating did not seal off the pores of the underlying gelatin carrier (Figure 2), the blood uptake was clearly compromised by the deposition of POx-NHS onto the patches. Hemopatch was included as well in these measurements, and showed significantly lower blood uptake values compared to G1-G3. Since blood uptake is necessary for satisfactory blood distribution throughout the patch and subsequent cross-linking, we concluded that $\mathbf{G} 3$ was the best-performing prototype in this test, as it allowed for more effective blood uptake compared to G4 and Hemopatch, but still prevented bleeding through the patches, which was observed for G1 and G2.

3.4.2. Adhesion Test. An in vitro adhesion test was performed (according to ASTM F2258-05 standards) to study the attachment between the coated patches upon contact with blood (Figure 3B). The different patches (G1-G4) were allowed to covalently cross-link onto each other for 1,5 , or 15 min, after which the adhesion force $(N)$ was measured until the patches were separated. Both a negative control (G1, carrier without polymer) and a benchmark (Hemopatch) were included in this study. The data demonstrated that the NHSester free blank samples (G1) did not adhere to each other, as reflected by adhesion forces of less than $0.5 \mathrm{~N}$, which confirms that NHS-ester groups are necessary for the formation of covalent cross-links. The coated samples (G2-G4) showed an entirely different behavior. At 1 and 5 min contacting time, low adhesion forces were measured that were comparable to G1, indicating a low degree of cross-linking. At $15 \mathrm{~min}$, however, a 3-fold larger force $(1.5 \mathrm{~N})$ was needed to separate both patches. This indicates that more cross-links are formed during the extended cross-linking time $(15 \mathrm{~min})$, resulting in larger adhesion forces. However, since these forces were in the same range for all three patches, it can be concluded that coating density did not affect the extent of adhesion in this experiment. By testing Hemopatch, adhesion forces within 1 and $5 \mathrm{~min}$ were similar to $\mathbf{G} \mathbf{2}-\mathbf{G} 4$ after $15 \mathrm{~min}$. We conclude that this product generally cross-links fast and forms strong gels with blood and carrier, which is in agreement with the blood gelation tests. While the differences regarding adhesion forces between the POx-NHS samples (G2-G4) and Hemopatch (15 $\mathrm{min}$ ) were statistically significant compared to NHS-ester free G1 (15 min), adhesion forces after 15 min were not statistically different between Hemopatch and the POx-NHS samples (G2-G4). In summary, it can be concluded that Hemopatch cross-linked faster than POx-NHS samples, whereas the final adhesion strength after $15 \mathrm{~min}$ was comparable for both samples.

3.5. In Vivo Efficacy Test. The POx functionalized patches were also evaluated in a clinically relevant setting by using an established in vivo pig model for profuse bleedings. ${ }^{40}$ In brief, standardized bleedings ( $8 \mathrm{~mm}$ diameter, $3 \mathrm{~mm}$ deep) were created in the liver and spleen of heparinized pigs $(n=4,30 \mathrm{~kg}$, $10 \mathrm{k}$ heparin). The bleedings were imaged at selected time points $(0,1$, and $5 \mathrm{~min}$ after creation of the bleeding) (Figure $4 \mathrm{~A})$ and the hemostatic efficacy of the different patches was assessed at 0,1 , and $5 \mathrm{~min}$ (bleeding/no bleeding). In addition, the bleeding score after 5 min was assessed using a visual scoring system ranging from 0 (effective hemostasis) to 4 (severe bleeding) (Figure 4B). The efficacy of hemostasis of POx-NHS coatings was tested for G3, which had a similar functional group density as the benchmark Hemopatch $(\sim 5.2$ mmol NHS $/ \mathrm{cm}^{2}$; Table 3$)$, but with a different polymer coating coverage. G1 was used as negative control (no coating). In addition, Tachosil (a collagen carrier coated with human derived fibrinogen and thrombin) was selected because of its common use during liver resections. ${ }^{15}$ The results of this study are depicted in Figure 4A,B. G3 was the best-performing hemostatic patch in this pig model; in 7 out of 8 events, hemostasis was obtained (bleeding score after $5 \mathrm{~min}$ : 0 , no bleeding; Figure 4B). In the remaining event ( 1 out of 8 ), insufficient pressure during application resulted in poor hemostatic action (bleeding score: 2, slight bleeding) (Figure 4B). In all cases, no significant blood flow through the patch was observed using G3, as was expected from the blood uptake experiments. Evidently, G1 was not effective at all in this bleeding model and significant blood flow through the patch was observed in line with the in vitro blood uptake experiments (Figure 3A). Moreover, in none of the events hemostasis was 
A
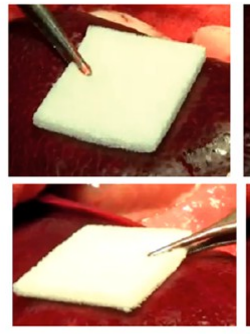

Hemopatch

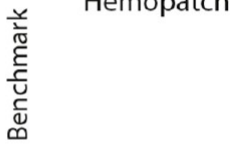

Tachosil

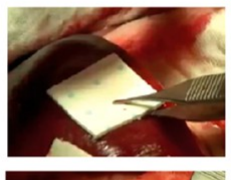

$\min$

1
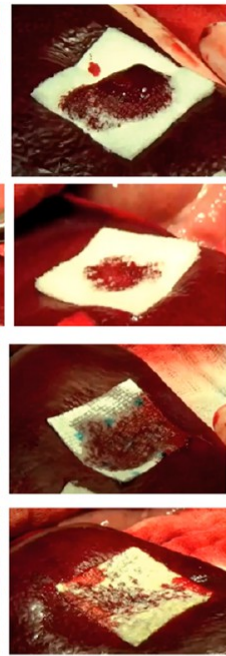

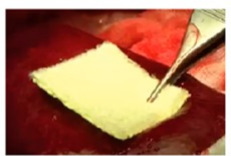

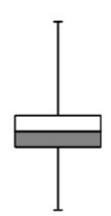

G3

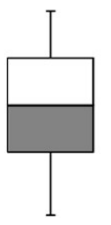

Hemopatch
Hemostasis

obtained

$0 / 4$

$7 / 8$

$5 / 8$

$2 / 8$

B
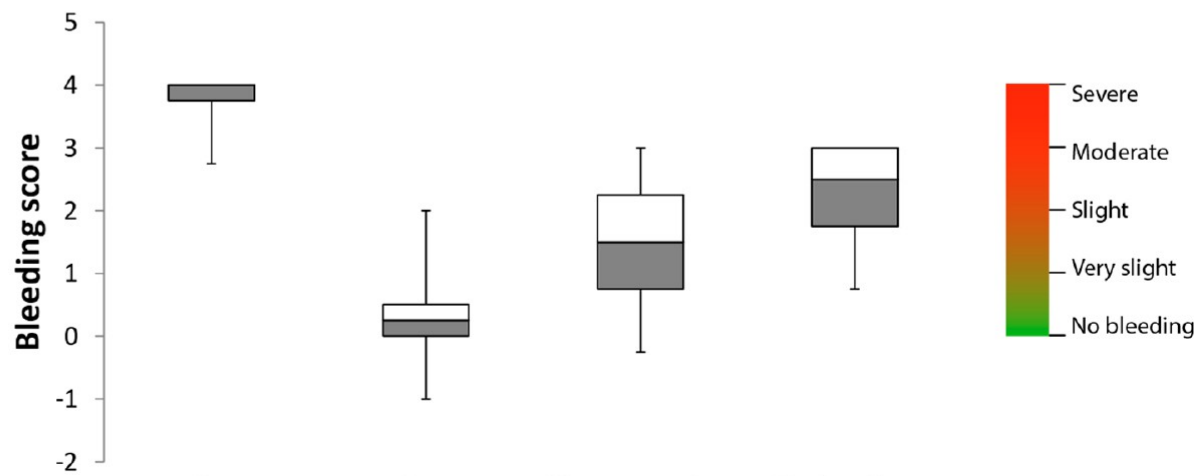

G1

0

6

Figure 4. In vivo study on pig spleen. (A) Images of the different prototypes at selected time points ( 0,1 , and 5 min), including the success rate of hemostasis. (B) Bleeding scores after 5 min according to the scoring system ${ }^{40}$

obtained, which can be related to the absence of chemical crosslinkers. As a result, using G1, in all events, severe bleedings were scored after 5 min (bleeding score: 4; Figure 4B). In the experiments using Tachosil, only in 2 out of 8 events hemostasis was observed, whereas moderate bleedings were scored for all other cases (Figure 4B). This poor hemostatic efficacy might be due to the use of a high heparin dose $(10 \mathrm{k}$ units) in this pig model, which inhibits hemostasis solely based on the natural coagulation cascade. Using Hemopatch, effective hemostasis was obtained in 5 out of 8 events (Figure 4A), where bleeding scores varied from 0 (no bleeding) to 3 (moderate bleedings; Figure 4B). Generally, Hemopatch adhered well and quickly to the tissue, which made repositioning challenging, a trend that was observed in the in vitro gelation tests as well. Unlike G3, slight bleeding at the edges of the patch was observed in cases where hemostasis was not obtained (Figure 4), which is possibly related to the inhomogeneous deposition of the polymer coating compared to the POx-NHS coated patches (Figure 2). From this in vivo study, it can be concluded that sealants that rely on chemical cross-linking with surrounding soft tissues and blood proteins (G3 and Hemopatch) hold great promise for the treatment of profuse bleeding models, unlike patches which are solely dependent on the natural coagulation cascade (noncoated patch (G1) and Tachosil), which were not effective in obtaining hemostasis in these models. Comparing G3 and Hemopatch, POx-NHS samples have the additional benefit that they are coated more homogeneously than Hemopatch, which results in a uniform sealing of the wound site. In addition, POxNHS samples are easier to handle due to their slower adhesion, which allows repositioning of the patch if required.

\section{CONCLUSIONS}

In this work, we have successfully developed a hemostatic device based on NHS-ester functionalized POx coated on a gelatin patch. We observed that the polymer should contain both NHS-esters as well as hydrophilic groups to ensure optimal hemostatic performance. Furthermore, we found that coating homogeneity and density are crucial parameters in order achieve the desired hemostatic action in vitro (measured by adhesion tests) as well as the desired amount of blood uptake. In vivo efficacy tests in a compromised pig model using heparin demonstrated that POx-NHS coated patches displayed a similar hemostatic efficacy as compared to Hemopatch. POxNHS patches were superior to products relying on activation of 
the natural coagulation cascade. In contrast to PEG, the structural versatility of $\mathrm{POx}$ allows further fine-tuning of the hemostatic performance, thereby rendering POx-NHS polymers excellent candidates for further development of hemostatic patches.

\section{ASSOCIATED CONTENT}

\section{S Supporting Information}

The Supporting Information is available free of charge on the ACS Publications website at DOI: 10.1021/acs.biomac.7b00683.

Description of the materials which are used and the experimental procedures of the monomers, intermediate products, and final polymers $(\mathbf{P 1}-\mathbf{P} 7)$ are described in S1. ${ }^{1} \mathrm{H}$ NMR spectra of both P3-P6 and P7, can be found in S2 and S3, respectively (PDF).

\section{AUTHOR INFORMATION}

\section{Corresponding Author}

*E-mail: j.vanhest@science.ru.nl; j.c.m.v.hest@tue.nl. ORCID ${ }^{\circ}$

Richard Hoogenboom: 0000-0001-7398-2058

Jan C. M. Van Hest: 0000-0001-7973-2404

Notes

The authors declare no competing financial interest.

\section{ACKNOWLEDGMENTS}

This work was supported by The Netherlands Institute for Regenerative Medicine (NIRM, Grant No. FES0908), NWO (KIEM 731.013.107, LIFT 731.015.415), Europees Fonds voor Regionale Ontwikkeling (EFRO 2011-014237) and GATT Technologies bv. The authors would like to thank Elvy de Hoog (Radboud University) for help with the polymer synthesis, Paul Riedel (Rubroeder $\mathrm{GmbH}$ ) for assisting with the spraying experiments, Roger Lomme (Department of Surgery (Radboud University medical Center) for assisting with the adhesion experiments and in vivo study, and Els van der Leyden (Ghent University) for the assisting with the contact angle measurements.

\section{REFERENCES}

(1) Marietta, M.; Facchini, L.; Pedrazzi, P.; Busani, S.; Torelli, G. Pathophysiology of bleeding in surgery. Transplant. Proc. 2006, 38 (3), $812-4$.

(2) Janssen, P. F.; Brölmann, H. A. M.; Huirne, J. A. F. Effectiveness of electrothermal bipolar vessel-sealing devices versus other electrothermal and ultrasonic devices for abdominal surgical hemostasis: a systematic review. Surg. Endosc. 2012, 26 (10), 2892-2901.

(3) Siegal, R. J.; Vaezy, S.; Martin, R.; Crum, L. Therapeutic Ultrasound, Part II* High Intensity Focused Ultrasound: A Method of Hemostasis. Echocardiography 2001, 18 (4), 309-315.

(4) Brustia, R.; Granger, B.; Scatton, O. An update on topical haemostatic agents in liver surgery: systematic review and meta analysis. J. Hepatobiliary. Pancreat. Sci. 2016, 23 (10), 609-621.

(5) Kim, F. J.; Rha, K. H.; Hernandez, F.; Jarrett, T. W.; Pinto, P. A.; Kavoussi, L. R. Laparoscopic radical versus partial nephrectomy: assessment of complications. J. Urol. 2003, 170 (2), 408-11.

(6) Tomizawa, Y. Clinical benefits and risk analysis of topical hemostats: a review. J. Artif. Organs 2005, 8 (3), 137-42.

(7) Bouten, P. J. M.; Zonjee, M.; Bender, J.; Yauw, S. T. K.; van Goor, H.; van Hest, J. C. M.; Hoogenboom, R. The chemistry of tissue adhesive materials. Prog. Polym. Sci. 2014, 39 (7), 1375-1405.
(8) Emilia, M.; Luca, S.; Francesca, B.; Luca, B.; Paolo, S.; Giuseppe, F.; Gianbattista, B.; Carmela, M.; Luigi, M.; Mauro, L. Topical hemostatic agents in surgical practice. Transfus. Apher. Sci. 2011, 45 (3), 305-11.

(9) Bruckner, B. A.; Blau, L. N.; Rodriguez, L.; Suarez, E. E.; Ngo, U. Q.; Reardon, M. J.; Loebe, M. Microporous polysaccharide hemosphere absorbable hemostat use in cardiothoracic surgical procedures. J. Cardiothorac. Surg. 2014, 9 (1), 1-7.

(10) Pusateri, A. E.; McCarthy, S. J.; Gregory, K. W.; Harris, R. A.; Cardenas, L.; McManus, A. T.; Goodwin, C. W., Jr. Effect of a chitosan-based hemostatic dressing on blood loss and survival in a model of severe venous hemorrhage and hepatic injury in swine. $J$. Trauma 2003, 54 (1), 177-82.

(11) Wu, Y.; He, J.; Cheng, W.; Gu, H.; Guo, Z.; Gao, S.; Huang, Y. Oxidized regenerated cellulose-based hemostat with microscopically gradient structure. Carbohydr. Polym. 2012, 88 (3), 1023-1032.

(12) Lewis, K. M.; Spazierer, D.; Urban, M. D.; Lin, L.; Redl, H.; Goppelt, A. Comparison of regenerated and non-regenerated oxidized cellulose hemostatic agents. Eur. Surg. 2013, 45, 213-220.

(13) Hajosch, R.; Suckfuell, M.; Oesser, S.; Ahlers, M.; Flechsenhar, K.; Schlosshauer, B. A novel gelatin sponge for accelerated hemostasis. J. Biomed. Mater. Res., Part B 2010, 94 (2), 372-9.

(14) Kabiri, M.; Emami, S. H.; Rafinia, M.; Tahriri, M. Preparation and characterization of absorbable hemostat crosslinked gelatin sponges for surgical applications. Curr. Appl. Phys. 2011, 11 (3), $457-461$.

(15) Moench, C.; Bechstein, W. O.; Hermanutz, V.; Hoexter, G.; Knaebel, H. P. Comparison of the collagen haemostat Sangustop(R) versus a carrier-bound fibrin sealant during liver resection; ESSCALIVER-Study. Trials 2010, 11, 109.

(16) Ghobril, C.; Grinstaff, M. W. The chemistry and engineering of polymeric hydrogel adhesives for wound closure: a tutorial. Chem. Soc. Rev. 2015, 44 (7), 1820-35.

(17) Leggat, P. A.; Smith, D. R.; Kedjarune, U. Surgical applications of cyanoacrylate adhesives: a review of toxicity. ANZ. J. Surg. 2007, 77 (4), 209-13.

(18) Öllinger, R.; Mihaljevic, A. L.; Schuhmacher, C.; Bektas, H.; Vondran, F.; Kleine, M.; Sainz-Barriga, M.; Weiss, S.; Knebel, P.; Pratschke, J.; Troisi, R. I. A multicentre, randomized clinical trial comparing the Veriset haemostatic patch with fibrin sealant for the management of bleeding during hepatic surgery. HPB 2013, 15 (7), $548-558$.

(19) Lewis, K. M.; Spazierer, D.; Slezak, P.; Baumgartner, B.; Regenbogen, J.; Gulle, H. Swelling, sealing, and hemostatic ability of a novel biomaterial: A polyethylene glycol-coated collagen pad. $J$. Biomater. Appl. 2014, 29 (5), 780-8.

(20) Lewis, K. M.; Kuntze, C. E.; Gulle, H. Control of bleeding in surgical procedures: critical appraisal of HEMOPATCH (Sealing Hemostat). Med. Devices: Evidence Res. 2015, 9, 1-10.

(21) Lewis, K. M.; Schiviz, A.; Hedrich, H. C.; Regenbogen, J.; Goppelt, A. Hemostatic efficacy of a novel, PEG-coated collagen pad in clinically relevant animal models. Int. J. Surg. 2014, 12 (9), 940-4.

(22) Howk, K.; Fortier, J.; Poston, R. A Novel Hemostatic Patch That Stops Bleeding in Cardiovascular and Peripheral Vascular Procedures. Ann. Vasc. Surg. 2016, 31, 186-195.

(23) Tomalia, D. A.; Sheetz, D. P. Homopolymerization of 2-alkyland 2-aryl-2-oxazolines. J. Polym. Sci., Part A-1: Polym. Chem. 1966, 4 (9), 2253-2265.

(24) Seeliger, W.; Aufderhaar, E.; Diepers, W.; Feinauer, R.; Nehring, R.; Thier, W.; Hellmann, H. Recent syntheses and reactions of cyclic imidic esters. Angew. Chem., Int. Ed. Engl. 1966, 5, 875-888.

(25) Levy, A.; Litt, M. Polymerization of cyclic iminoethers. V. 1,3oxazolines with hydroxy-, acetoxy-, and carboxymethyl-alkyl groups in the 2 position and their polymers. J. Polym. Sci., Part A-1: Polym. Chem. 1968, 6 (7), 1883-1894.

(26) Kagiya, T.; Narisawa, S.; Maeda, T.; Fukui, K. Ring-opening polymerization of 2-substituted 2-oxazolines. J. Polym. Sci., Part B: Polym. Lett. 1966, 4 (7), 441-445. 
(27) Hoogenboom, R. Poly(2-oxazoline)s: a polymer class with numerous potential applications. Angew. Chem., Int. Ed. 2009, 48 (43), 7978-94.

(28) Rossegger, E.; Schenk, V.; Wiesbrock, F. Design Strategies for Functionalized Poly(2-oxazoline)s and Derived Materials. Polymers 2013, 5 (3), 956-1011.

(29) Guillerm, B.; Monge, S.; Lapinte, V.; Robin, J. J. How to modulate the chemical structure of polyoxazolines by appropriate functionalization. Macromol. Rapid Commun. 2012, 33 (19), 1600-12.

(30) Kronek, J.; Paulovičová, E.; Paulovičová, L.; Kroneková, Z.; Lustoň, J. Immunomodulatory efficiency of poly(2-oxazolines). J. Mater. Sci.: Mater. Med. 2012, 23 (6), 1457-1464.

(31) Bauer, M.; Lautenschlaeger, C.; Kempe, K.; Tauhardt, L.; Schubert, U. S.; Fischer, D. Poly(2-ethyl-2-oxazoline) as alternative for the stealth polymer poly(ethylene glycol): comparison of in vitro cytotoxicity and hemocompatibility. Macromol. Biosci. 2012, 12 (7), 986-98.

(32) Adams, N.; Schubert, U. S. Poly(2-oxazolines) in biological and biomedical application contexts. Adv. Drug Delivery Rev. 2007, 59 (15), 1504-20.

(33) Gaertner, F. C.; Luxenhofer, R.; Blechert, B.; Jordan, R.; Essler, M. Synthesis, biodistribution and excretion of radiolabeled poly(2alkyl-2-oxazoline)s. J. Controlled Release 2007, 119 (3), 291-300.

(34) Wyffels, L.; Verbrugghen, T.; Monnery, B. D.; Glassner, M.; Stroobants, S.; Hoogenboom, R; Staelens, S. muPET imaging of the pharmacokinetic behavior of medium and high molar mass (89) Zrlabeled poly(2-ethyl-2-oxazoline) in comparison to poly(ethylene glycol). J. Controlled Release 2016, 235, 63-71.

(35) de la Rosa, V. R. Poly(2-oxazoline)s as materials for biomedical applications. J. Mater. Sci.: Mater. Med. 2014, 25 (5), 1211-25.

(36) Moreadith, R. W.; Viegas, T. X.; Bentley, M. D.; Harris, J. M.; Fang, Z.; Yoon, K.; Dizman, B.; Weimer, R.; Rae, B. P.; Li, X.; Rader, C.; Standaert, D.; Olanow, W. Clinical development of a poly(2oxazoline) (POZ) polymer therapeutic for the treatment of Parkinson's disease - Proof of concept of $\mathrm{POZ}$ as a versatile polymer platform for drug development in multiple therapeutic indications. Eur. Polym. J. 2017, 88, 524.

(37) Zalipsky, S.; Hansen, C. B.; Oaks, J. M.; Allen, T. M. Evaluation of Blood Clearance Rates and Biodistribution of Poly(2-oxazoline)Grafted Liposomes§. J. Pharm. Sci. 1996, 85 (2), 133-137.

(38) Lih, E.; Lee, J. S.; Park, K. M.; Park, K. D. Rapidly curable chitosan-PEG hydrogels as tissue adhesives for hemostasis and wound healing. Acta Biomater. 2012, 8 (9), 3261-9.

(39) ASTM F2258-05(2015), Standard Test Method for Strength Properties of Tissue Adhesives in Tension; ASTM International: West Conshohocken, PA, 2015, www.astm.org. In ASTM F2258-05(2015), Standard Test Method for Strength Properties of Tissue Adhesives in Tension, ASTM International, West Conshohocken, PA, 2015, www. astm.org.

(40) Adams, G. L.; Manson, R. J.; Hasselblad, V.; Shaw, L. K.; Lawson, J. H. Acute in-vivo evaluation of bleeding with Gelfoam plus saline and Gelfoam plus human thrombin using a liver square lesion model in swine. J. Thromb. Thrombolysis 2009, 28 (1), 1-5.

(41) Mees, M. A.; Hoogenboom, R. Functional Poly(2-oxazoline)s by Direct Amidation of Methyl Ester Side Chains. Macromolecules 2015, 48 (11), 3531-3538.

(42) Rueda, J. C.; Campos, E.; Komber, H.; Zschoche, S.; Häussler, L.; Voit, B. Synthesis and characterization of new $\mathrm{pH}-$ and thermoresponsive hydrogels based on $\mathrm{N}$-isopropylacrylamide and 2-oxazolines. Des. Monomers Polym. 2014, 17 (3), 208-216.

(43) Rueda, J. C.; Asmad, M.; Ruiz, V.; Komber, H.; Zschoche, S.; Voit, B. Synthesis and characterization of new bi-sensitive copoly(2oxazolines). Des. Monomers Polym. 2015, 18 (8), 761-769.

(44) Boerman, M. A.; Van der Laan, H. L.; Bender, J. C. M. E.; Hoogenboom, R.; Jansen, J. A.; Leeuwenburgh, S. C.; Van Hest, J. C. M. Synthesis of $\mathrm{pH}$ - and thermoresponsive poly(2-n-propyl-2oxazoline) based copolymers. J. Polym. Sci., Part A: Polym. Chem. 2016, 54 (11), 1573-1582.
(45) Bouten, P. J. M.; Hertsen, D.; Vergaelen, M.; Monnery, B. D.; Boerman, M. A.; Goossens, H.; Catak, S.; van Hest, J. C. M.; Van Speybroeck, V.; Hoogenboom, R. Accelerated living cationic ringopening polymerization of a methyl ester functionalized 2-oxazoline monomer. Polym. Chem. 2015, 6 (4), 514-518.

(46) Bouten, P. J. M.; Hertsen, D.; Vergaelen, M.; Monnery, B. D.; Catak, S.; van Hest, J. C. M.; Van Speybroeck, V.; Hoogenboom, R. Synthesis of poly(2-oxazoline)s with side chain methyl ester functionalities: Detailed understanding of living copolymerization behavior of methyl ester containing monomers with 2-alkyl-2oxazolines. J. Polym. Sci., Part A: Polym. Chem. 2015, 53 (22), 2649-2661.

(47) Wiesbrock, F.; Hoogenboom, R.; Leenen, M. A. M.; Meier, M. A. R; Schubert, U. S. Investigation of the Living Cationic RingOpening Polymerization of 2-Methyl-, 2-Ethyl-, 2-Nonyl-, and 2Phenyl-2-oxazoline in a Single-Mode Microwave Reactor $\dagger$. Macromolecules 2005, 38 (12), 5025-5034.

(48) Wijnans, S.; de Gans, B.-J.; Wiesbrock, F.; Hoogenboom, R.; Schubert, U. S. Characterization of a Poly(2-oxazoline) Library by High-Throughput, Automated Contact-Angle Measurements and Surface-Energy Calculations. Macromol. Rapid Commun. 2004, 25 (23), 1958-1962. 